\title{
Correction to: Infinitary Addition, Real Numbers, and Taut Monads
}

\author{
George Janelidze ${ }^{1} \cdot$ Ross Street $^{2}$
}

Published online: 12 May 2018

(c) Springer Science+Business Media B.V., part of Springer Nature 2018

\section{Correction to: Appl Categor Struct https://doi.org/10.1007/s10485-018-9524-4}

In the original publication of the article, Eq. (3.24) was published incorrectly. The corrected equation is given in this correction article.

$$
\begin{aligned}
& a_{0}=\Sigma_{i \in \mathbb{N}} b_{i} \Rightarrow \\
& \quad\left(\left(\exists_{a_{1}, a_{2}, \ldots} \forall_{n \in \mathbb{N}} a_{n}=b_{n}+a_{n+1}\right) \& \forall_{c_{0}, c_{1}, \ldots}\left(\left(\forall_{n \in \mathbb{N}} c_{n}=b_{n}+c_{n+1}\right) \Rightarrow a_{0} \leq c_{0}\right)\right),
\end{aligned}
$$

The original article has been corrected.

The original article can be found online at https://doi.org/10.1007/s10485-018-9524-4.

$凶$ George Janelidze

George.Janelidze@uct.ac.za

Ross Street

ross.street@mq.edu.au

1 Department of Mathematics and Applied Mathematics, University of Cape Town, Rondebosch 7701, South Africa

2 Mathematics Department, Macquarie University, Sydney, NSW 2109, Australia 